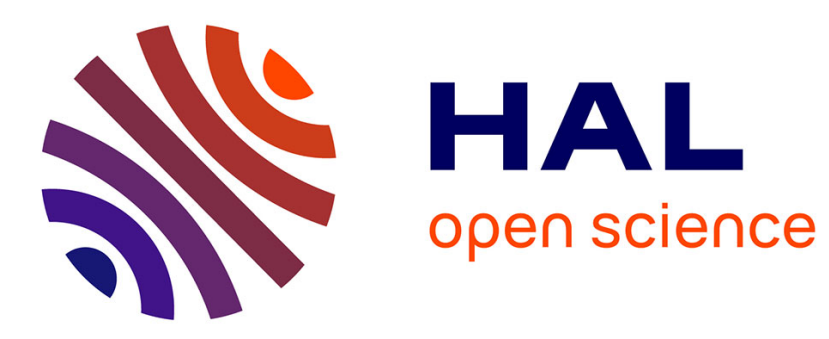

\title{
A Statistics-Based Definition of the Overmoded Condition for Reverberation Chambers Andrea Cozza
}

\section{To cite this version:}

Andrea Cozza. A Statistics-Based Definition of the Overmoded Condition for Reverberation Chambers. XXX General Assembly and Scientific Symposium of the International Union of Radio Science, Aug 2011, Istanbul, Turkey. pp.1. hal-00587446

\section{HAL Id: hal-00587446 https://hal.science/hal-00587446}

Submitted on 20 Apr 2011

HAL is a multi-disciplinary open access archive for the deposit and dissemination of scientific research documents, whether they are published or not. The documents may come from teaching and research institutions in France or abroad, or from public or private research centers.
L'archive ouverte pluridisciplinaire HAL, est destinée au dépôt et à la diffusion de documents scientifiques de niveau recherche, publiés ou non, émanant des établissements d'enseignement et de recherche français ou étrangers, des laboratoires publics ou privés. 


\title{
A Statistics-Based Definition of the Overmoded Condition for Reverberation Chambers
}

\author{
Andrea Cozza \\ Département de Recherche en Électromagnétisme, SUPELEC \\ 3 rue Joliot-Curie, 91192 Gif-sur-Yvette, France \\ andrea.cozza@supelec.fr
}

\begin{abstract}
Current understanding of reverberation chambers is firmly funded upon their being operated as overmoded cavities. A proper definition of this condition has been lacking for a long time, while being regarded as automatically satisfied as soon as a large (and unspecified) number of normal modes are set to resonate at the frequency of operation of the chamber. In this paper we summarize the most important steps needed to introduce in a formal way a proper definition of the overmoded condition, showing how, from a strict mathematical viewpoint, it is not related to any threshold frequency, but rather requires a statistical framework.
\end{abstract}

\section{Introduction}

Reverberation chambers (RCs) are electrically large closed cavities characterized by very weak dissipation phenomena over its boundaries (walls) and within their volume. Apart as a fundamental tool for experimental investigations in quantum chaos and other fundamental research topics, RCs are widely used as tests facilities in Electromagnetic Compatibility (EMC), mostly in the case of radiated tests. The interested Reader can refer to [1, 2] as a useful introduction on the way RCs are actually utilized in EMC and their physics. The topic of concern in this paper is rather the fact that their use is based on a strong assumption, that they are capable of providing a diffused field. This property is never directly invoked in RC theory as developed within the EMC community, though it is of fundamental importance in the physics of cavities and the study of room acoustics [3]. Historical reasons are likely to blame for this mismatch, as well as the fact that electrical engineers have long preferred to have a more practical and test-driven approach to the understanding of these facilities. Still, the lack of the clear definition of a diffused field has been an invisible obstacle, in our opinion, to the cross-fertilization that should have existed between the EMC community and the acoustics one. Indeed, EMC engineers could benefit from the deeper understanding and modeling tools developed in acoustics. In this respect, we will regard the concepts of overmoded RC and that of diffused field as synonyms, as both refer to a cavity that ensures, asymptotically, to expect a very specific statistical behavior for field-related quantities.

Yet, both fields lacked a proper physics-based model capable of explaining under what conditions a cavity can be considered to allow a diffused field to establish. Acoustics, too, tends to consider the idea of a threshold frequency [3], often referred to as Lowest Usable Frequency (LUF) in EMC. In a recent paper [4], we have proposed such a model, establishing a direct link between the physics of an RC and the statistical properties of the field it supports. In this paper we recall the most important steps and assumptions that have led to this result, and show how it can explain another phenomenon with an unsatisfying description, that of local statistical non-compliancy of field-related quantities. Our definition of an ovemoded cavity (and thus of a diffused field) is based on the use of a modal description of the field excited within an RC, coupled to a statistical approach. The interest of this approach is that it allows avoiding making non-physical assumptions on the need for a threshold on the modal density, as apparently assumed on the EMC side, or on the sheer fact that a threshold frequency exists. Of particular importance is the prediction that losses should not be regarded as a limitation or nuisance in the operation of RCs, but that they rather lie at the heart of its functioning. Without losses, an RC would never be capable of supporting a diffused field, independently of the modal density it supports: hence, our regarding the use of the term overmoded as a potentially treacherous habit, as the mere availability of a high modal density is not a sufficient condition. In the following, it will be shown that an overmoded $\mathrm{RC}$ is a cavity that supports a large number of overlapped modes, which is not the same as just supporting a large number of modes, as estimated by looking at modal density alone. This fact was unacknowledged in EMC, while well understood in acoustics: still, both have always considered that the idea of a threshold frequency be reasonable, a fact that is disproved in this paper. 


\section{The overmoded condition as a statistical asymptotic condition}

The common sense given to the concept of overmoded cavity is tightly linked to the fulfillment of the following properties: 1) field statistics are independent of the position in space where they are tested, at least in a sub-volume of the cavity; 2) the three orthogonal components of the electric (respectively, magnetic) field behave as independent and identically (iid) distributed random variables; 3 ) they follow a Gaussian distribution law, with zero mean-value. It has been shown that these hypothesis are met whenever the field distribution can be described by means of a continuous random plane-wave spectrum, with specific statistical properties [5]. Although such type of model well approximates the behavior of real-life RCs in their high-frequency range, it is well-known that there might exist some frequencies where it is incapable of predicting a drift from it [6]. These disagreements are often explained through a stirrer inefficiency or a sub-optimal alignment for the sources [7], though we are quite skeptical of these explanations [8], whereas in the lower frequency range they are usually just fitted to alternative probability distribution functions [9].

It is, in our opinion, by far more interesting and useful to get back to the basics of the physics of cavities, while taking on a more statistical approach from the beginning. To this end, we will consider a modal expansion for the electric field distribution observed at the position $r$ within an RC at the working frequency $f$ [10]

$$
\boldsymbol{E}(\boldsymbol{r}, f)=\sum_{i \in \mathscr{M}} \gamma_{i}(\boldsymbol{r}, f) \psi_{i}(f) \hat{\boldsymbol{\xi}}_{i}(\boldsymbol{r}, f)
$$

where the $\gamma_{i}(\boldsymbol{r}, f)$ reflect the excitation of each mode, depending on the nature of the source and its position relative to the modal topographies, $\psi_{i}(f)$ is the bell-shaped frequency response of each mode and $\hat{\boldsymbol{\xi}}_{i}(\boldsymbol{r}, f)$ is the polarization of the electric field contribution provided by each mode. The sum in (1) is taken over the set $\mathscr{M}$ of modes that are effectively excited at the working frequency. A proper justification for this model has been provided in [4]. Rather than attempting to apply (1) in a deterministic way, we will regard all of these modal quantities as random ones, with statistical properties dictated (or suggested) by their physical properties.

In this respect, the modal weights $\left\{\gamma_{i}\right\}$ can be expected to behave as centered random variables, as they are directly related to the scalar projection of the equivalent currents representing a source and the modal topographies, which are standing waves, thus characterized by a pseudo-periodic change in their sign over space. No further assumption is needed on the nature of the probability law for the $\gamma_{i}$. The functions $\left\{\psi_{i}(f)\right\}$ represent the responses of second-order systems, defined as follows

$$
\psi_{i}(f)=\frac{1}{f_{i}^{2}\left(1+\mathrm{j} / 2 Q_{i}\right)^{2}-f^{2}},
$$

where $f_{i}$ is the resonance frequency of the $i$-th mode and $Q_{i}$ its quality factor. As the presence of a stirring technique implies, from an ideal perspective, that the frequencies of resonance of the modes are thoroughly mixed and swapped about the working frequency, it is reasonable to assume that the variables $\left\{f_{i}\right\}$ be uniformly distributed. Moreover, the quality factors $\left\{Q_{i}\right\}$ can be characterized by means of their average (or composite) value, as will be shown later. This latter quantity can be experimentally assessed. Finally, the polarization unit vectors $\left\{\hat{\boldsymbol{\xi}}_{i}\right\}$ will be assumed to be uniformly distributed over $4 \pi$ steradian, again as a consequence of the assumption of an ideal stirring technique at work. It is important to notice that assuming a perfect stirring is actually sensible, as we are interested in assessing whether it is possible to explain the eventual non-compliancy of an RC even having enforced an ideal performance on the stirring technique.

With this model at our disposal, we now aim at studying the first two statistical moments of the electric energy density

$$
W(\boldsymbol{r}, f)=\epsilon_{0}\|\boldsymbol{E}(\boldsymbol{r}, f)\|^{2},
$$

with $\epsilon_{0}$ the dielectric permittivity of the medium filling the cavity. The electric energy density can be described, in the case of an ideally diffused field, as a random variable distributed as a $\chi_{6}^{2}$ law, as a consequence of the features recalled at the beginning of this Section. As opposed to this hypothesis, by computing the first two moments of $W(\boldsymbol{r}, f)$ and employing the previously introduced assumptions on the statistics of the modal quantities should allow to check under what conditions the ideal behavior is actually met. The procedure for this calculation has been presented in details 


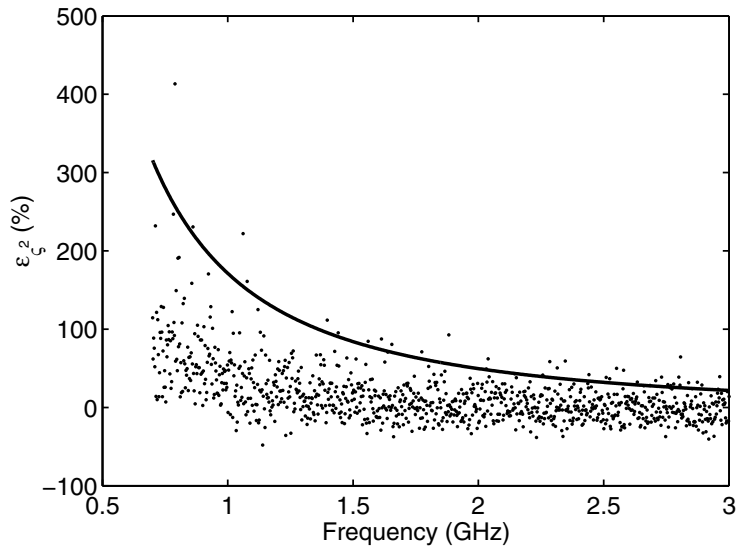

(a)

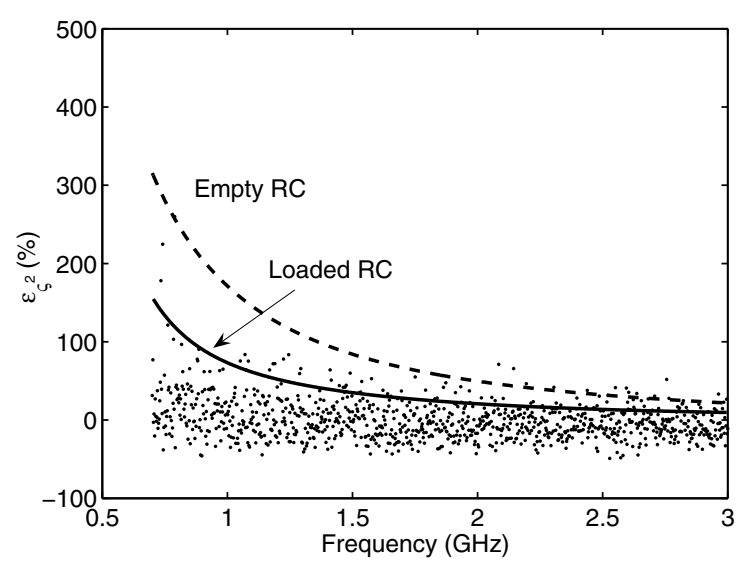

(b)

Figure 1: Estimates of the standardized variance of the energy density $W$, as assessed from experimental results obtained for : (a) an empty cavity (apart for the excitation antenna) and (b) one loaded with a set of 4 pyramidal absorbers about $30 \mathrm{~cm}$ high.

in [4], and leads to the following standardized variance

$$
\varsigma_{W}^{2}=\left(\frac{\sigma_{W}}{\mu_{W}}\right)^{2}=\frac{1}{3}+\frac{1}{\pi} \frac{\mu_{4}}{\mu_{2}^{2}} \frac{1}{M_{M}},
$$

with $M_{M}=m B_{M}$ the average number of modes overlapping within the average $-3 \mathrm{~dB}$ bandwidth $B_{M}=f / Q$ centered around the working frequency, for a modal density $m(f)$ and an average composite quality factor $Q$. The terms $\mu_{n}$ refer to the moments of the modal weights $\left\{\gamma_{i}\right\}$

$$
\mu_{n}=\mathrm{E}\left[\left|\gamma_{i}\right|^{n}\right],
$$

so that $\mu_{4} / \mu_{2}^{2}=2$ for the case of normally distributed modal weights, which is the usual assumption applied for these quantities. Other distribution laws do not have much of an effect on this ratio, so that the final result is weakly dependent on any assumption on the nature of the modal weights.

Comparing (4) to the standardized variance $\varsigma_{\chi_{6}^{2}}^{2}=1 / 3$ obtained for the ideal diffused field scenario shows what is needed for an $\mathrm{RC}$ to be as close as possible to the ideal case, i.e., presenting a large number of overlapped modes $M_{M}$. Whence, it is clear that there is no reason for assuming that any threshold frequency ensures an RC to support a diffused field, as $M_{M}$ is actually a complex function of frequency, with a non monotonous trend. As a matter of fact, both the composite quality factor $Q$ and the modal density $m$ are non-monotonous; attention must be paid to the fact that the often used Weyl's approximation is indeed a smooth fitting curve to the real $m(f)$, which is generally not known. Local variations around this smooth function can be far from negligible, leading to modal depletion or excess: it can therefore be intuitively understood that $M_{M}$ follows a similar trend, whose range of variations is made further wider and unpredictable by the dominant role of the composite quality factor $Q$.

As a result, the standardized variance $\varsigma_{W}^{2}$ can get quite larger than the $1 / 3$ value expected in the ideal case. This value is attained only asymptotically as $M_{M} \rightarrow \infty$, a condition that can be thought as well approximated as the working frequency increases, since the modal density will increase (on average) quadratically with the frequency, while the composite quality factor has typically a slower rate of growth. In any case, (4) links the rate of convergence of the statistics of $W$ to the average number of overlapped modes $M_{M}$ : hence, by settling on an acceptable error between the real and ideal $\varsigma^{2}$, we can come up with a threshold on the minimum number of modes that should overlap on each other bandwidth, as a way to ensure this maximum error.

Since $M_{M}(f)$ can be reasonably expected to broadly increase with the frequency, (4) predicts that the standardized variance of the energy density should converge to the asymptotic value $1 / 3$ at high frequency. This fact is demonstrated 
in Fig. 1a, where experimentally determined estimates of $\varsigma_{W}^{2}$ are shown over a wide frequency range, as obtained with a 100-step mechanical stirrer in an $\mathrm{RC}$ with a volume of $13.8 \mathrm{~m}^{3}$. The RC was excited by means of a log-periodic dipole antenna pointed at one corner, while the field samples were measured with an electro-optical probe positioned at the center of the cavity. Fig. 1 confirms that indeed the relative error $\epsilon_{\varsigma^{2}}=\left(\varsigma_{W}^{2}-1 / 3\right) /(1 / 3)$ broadly decreases with the frequency, while it is far from negligible in the lower frequency range. The thicker line in Fig. 1 has been obtained by applying (4), using a loose estimate for $M_{M}$. This latter quantity was derived by using the average modal density predicted by Weyl's formula and a smooth majorant of an experimentally-derived estimate of the composite quality factor. This operation was aimed at assessing whether (4) is capable of providing a loose upper-bound for the error observed in practice on the standardized variance $\varsigma_{W}^{2}$. Indeed, a good agreement between the prediction of the maximum error yielded by (4) and the experimental results is observed, with an envelop well identified by the former.

The case treated in Fig. 1b deals with the introduction of additional losses: in this case too our model predicts in a fairly good way the reduction of the variability of $W$. Further details about the validity of (4) are provided in [4], particularly about the role of losses in the statistical compliance of the energy density in an RC.

\section{Conclusions}

In this paper we have briefly recalled the reasons of our introducing a physically-motivated definition of the overmoded condition for an RC. The use of a modal approach, though inevitably based on simplifying assumptions, has led to pointing out that there is no threshold frequency for a real RC to behave as an ideal one. Local disagreements for the statistics of field-related quantities can appear at any frequency, depending on eventual configurations featuring a weak overlapping of modes. The probability for these events is intuitively expected to decrease for an increasing frequency, and the proposed model indeed supports this idea. Therefore, it is uncorrect to consider that departures from the asymptotic statistical behavior of the field statistics be due to an imperfect design of the RC setup, be it the design of the cavity excitation or the stirring technique.

\section{References}

[1] D. Hill, "Electromagnetic theory of reverberation chambers," NIST Technical note, no. 1506, 1998.

[2] Reverberation chamber test methods, International Electrotechnical Commission (IEC) Std. 61 000-4-21, 2003.

[3] M. Schroeder, "Statistical parameters of the frequency response curves of large rooms," J. Audio Eng. Soc, vol. 35, no. 5, pp. 299-305, 1987.

[4] A. Cozza, "The role of losses in the definition of the overmoded condition for reverberation chambers and their statistics," to appear in IEEE Transactions on Electromagnetic Compatibility.

[5] D. Hill, "Plane wave integral representation for fields in reverberation chambers," IEEE Transactions on Electromagnetic Compatibility, vol. 40, no. 3, pp. 209-217, 1998.

[6] V. Primiani, F. Moglie, and V. Paolella, "Numerical and experimental investigation of unstirred frequencies in reverberation chambers," in Electromagnetic Compatibility, 2009. EMC 2009. IEEE International Symposium on, August 2009, pp. 177-181.

[7] O. Lundén and M. Bäckström, "How to avoid unstirred high frequency components in mode stirred reverberation chambers," in Electromagnetic Compatibility, 2007. EMC 2007. IEEE International Symposium on, July 2007, pp. 1-4.

[8] A. Cozza, “A skeptic's view of unstirred components," in submitted to EMC Europe 2011, 2011.

[9] G. Orjubin, E. Richalot, S. Mengue, and O. Picon, "Statistical model of an undermoded reverberation chamber," Electromagnetic Compatibility, IEEE Transactions on, vol. 48, no. 1, pp. 248 -251, feb. 2006.

[10] J. Van Bladel, Electromagnetic fields. IEEE, 2007. 